\title{
Some limit theorems for the second-order Markov chains indexed by a general infinite tree with uniform bounded degree
}

\author{
Zhiyan Shi ${ }^{{ }^{*}}$, Weiguo Yang ${ }^{1}$, Lixin $\operatorname{Tian}^{1}$ and Qingpei Zang ${ }^{1,2}$
}

\author{
* Correspondence: \\ shizhiyan1984@126.com \\ ${ }^{1}$ Faculty of Science, Jiangsu \\ University, Zhenjiang, 212013, \\ China \\ Full list of author information is \\ available at the end of the article
}

\begin{abstract}
In this paper, we study a convergence theorem for a finite second-order Markov chain indexed by a general infinite tree with uniformly bounded degree. Meanwhile, the strong law of large numbers (LLN) and Shannon-McMillan theorem for a finite second-order Markov chain indexed by this tree are obtained. 2000 Mathematics Subject Classification: 60F15; 60J10.

Keywords: uniformly bounded tree, second-order Markov chain, strong law of large numbers, Shannon-McMillan theorem
\end{abstract}

\section{Introduction}

A tree is a graph $G=\{T, E\}$ that is connected and contains no circuits. Given any two vertices $\sigma, t(\sigma \neq t \in T)$, let $\overline{\sigma t}$ be the unique path connecting $\sigma$ and $t$. Define the graph distance $d(\sigma, t)$ to be the number of edges contained in the path $\overline{\sigma t}$.

Let $T_{C, N}$ be a Cayley tree. In this tree, the root (denoted by $o$ ) has only $N$ neighbors, and all other vertices have $N+1$ neighbors. Let $T_{B, N}$ be a Bethe tree, on which each vertex has $N+1$ neighboring vertices. Here, both $T_{C, N}$ and $T_{B, N}$ are homogeneous tree. An infinite tree with uniformly bounded degree is that the numbers of neighbors of any vertices in this tree are uniformly bounded. Therefore, the homogeneous tree is the special case of infinite tree with uniformly bounded degree. In this paper, we mainly consider a general infinite tree with uniformly bounded degree, that is, the tree is formed by an infinite tree that has uniformly bounded degree with the root $o$ connecting with another point denoted by the root -1 . In order to understand this tree graph, we give a figure (see Figure 1), which is formed by a $T_{C, 2}$ with the root $o$ connecting with another root -1 . When the context permits, this type of trees is all denoted simply by $T$.

Let $\sigma, t(\sigma, t \neq o,-1)$ be vertices of a general infinite tree with uniformly bounded degree $T$. Write $t \leq \sigma$ if $t$ is on the unique path connecting $o$ to $\sigma$, and $|\sigma|$ for the number of edges on this path. For any two vertices $\sigma, t(\sigma, t \neq o,-1)$ of tree $T$, denote by $\sigma \wedge t$ the vertex farthest from $o$ satisfying $\sigma \wedge t \leq \sigma$ and $\sigma \wedge t \leq t$.

The set of all vertices with distance $n$ from root $o$ is called the $n$th generation of $T$, which is denoted by $L_{n}$. We say that $L_{n}$ is the set of all vertices on level $n$ and especially root-1 is on the -1 st level on tree $T$. We denote by $T^{(n)}$ the subtree of a general

(c) 2012 Shi et al; licensee Springer. This is an Open Access article distributed under the terms of the Creative Commons Attribution License (http://creativecommons.org/licenses/by/2.0), which permits unrestricted use, distribution, and reproduction in any medium, provided the original work is properly cited. 


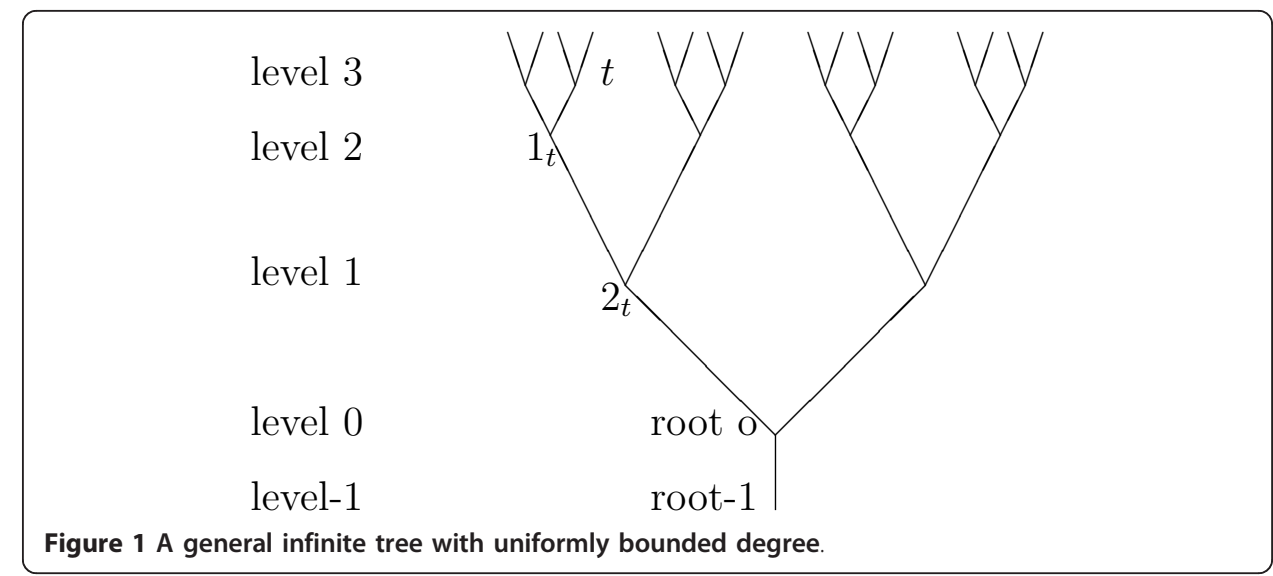

infinite tree with uniformly bounded degree $T$ containing the vertices from level -1 (the root -1$)$ to level $n$. Let $t(\neq o,-1)$ be a vertex of a general infinite tree with uniformly bounded degree $T$. Predecessor of the vertex $t$ is another vertex that is nearest from $t$ on the unique path from root -1 to $t$. We denote the predecessor of $\mathrm{t}$ by $1_{t}$ and the predecessor of $1_{t}$ by $2_{t}$. We also say that $2_{t}$ is the second predecessor of $t . X^{A}=$ $\left\{X_{t}, t \in A\right\}$ and denoted by $|A|$ the number of vertices of $A$, and $x^{A}$ is the realization of $X^{A}$.

Definition 1 Let $G=\{1,2, \ldots, N\}$ and $P(z \mid y, x)$ be a nonnegative functions on $G^{3}$. Let

$$
P=(P(z \mid y, x)), \quad P(z \mid y, x) \geq 0, x, y, z \in G \text {. }
$$

If

$$
\sum_{z \in G} P(z \mid y, x)=1
$$

$P$ will be called a second-order transition matrix.

Definition 2 Let $T$ be a general infinite tree, $G=\{1,2, \ldots, N\}$ be a finite state space, and $\left\{X_{t}, t \in T\right\}$ be a collection of $G$-valued random variables defined on the probability space $(\Omega, \mathcal{F}, P)$. Let

$$
P=(p(x, y)), \quad x, y \in G
$$

be a distribution on $G^{2}$, and

$$
P=(P(z \mid y, x)), \quad x, y, z \in G
$$

be a second-order transition matrix. If for any vertex $t(t \neq 0,-1)$,

$$
\begin{aligned}
& P\left(X_{t}=z \mid X_{1_{t}}=y, X_{2_{t}}=x, \text { and } X_{\sigma} \text { for } \sigma \wedge t \leq 1_{t}\right) \\
& =P\left(X_{t}=z \mid X_{1_{t}}=y, X_{2_{t}}=x\right)=P(z \mid y, x) \quad \forall x, y \in G,
\end{aligned}
$$

and

$$
P\left(X_{-1}=x, X_{o}=y\right)=p(x, y), \quad x, y \in G,
$$

$\left\{X_{t}, t \in T\right\}$ will be called a $G$-valued second-order Markov chain indexed by a general infinite tree $T$ with the initial distribution (1) and second-order transition matrix (2) or called a $T$-indexed second-order Markov chain. 
The subject of tree-indexed processes is rather young. Benjamini and Peres (see [1]) have given the notion of the tree-indexed Markov chains and studied the recurrence and ray-recurrence for them. Berger and Ye (see [2]) have studied the existence of entropy rate for some stationary random fields on a homogeneous tree. Ye and Berger (see [3,4]), by using Pemantle's result(see [5]) and a combinatorial approach, have studied the Shannon- McMillan theorem with convergence in probability for a PPG-invariant and ergodic random field on a homogeneous tree. Yang and Liu(see [6])have studied a strong law of large numbers for the frequency of occurrence of states for Markov chains field on a homogeneous tree (a particular case of tree-indexed Markov chains field and PPG-invariant random field). Yang (see [7]) has studied the strong law of large numbers for frequency of occurrence of state and Shannon-McMillan theorem for homogeneous Markov chains indexed by a homogeneous tree. Takacs (see [8]) has studied the strong law of large numbers for the univariate functions of finite Markov chains indexed by an infinite tree with uniformly bounded degree. Recently, Yang (see [9]) has studied the strong law of large numbers and Shannon-McMillan theorem for nonhomogeneous Markov chains indexed by a homogeneous tree. Huang and Yang (see [10]) have studied the strong law of large numbers for Markov chains indexed by an infinite tree with uniformly bounded degree, which generalize the result of [8]. Shi and Yang (see [11]) have also studied a limit property of random transition probability for a second-order nonhomogeneous Markov chains indexed by a tree.

In this paper, we first study a convergence theorem for a finite second-order Markov chain indexed by a general infinite tree with uniformly bounded degree. As corollaries, we obtain some limit theorems for the frequencies of occurrence of states for this Markov chain. Finally, we obtain the strong law of large numbers and ShannonMcMillan theorem for a class of finite second-order Markov chain indexed by a general infinite tree with uniformly bounded degree.

\section{Strong limit theorems}

Lemma 1 Let $T$ be a general infinite tree with uniformly bounded degree. Let $\left\{X_{t}, t \in\right.$ $T\}$ be a $T$-indexed second-order Markov chain with state space $G$ defined as in Definition 2, $\left\{g_{t}(x, y, z), t \in T\right\}$ be a collection of functions defined on $G^{3}$. Let $L_{-1}=\{-1\}, L_{0}$ $=\{0\}$ and $\mathcal{F}_{n}=\sigma\left(X^{T^{(n)}}\right)(n \geq 1)$. Set

$$
F_{n}(\omega)=\sum_{t \in T^{(n)} \backslash\{0\}\{-1\}} g_{t}\left(X_{2_{t}}, X_{1_{t}}, X_{t}\right)
$$

and

$$
t_{n}(\lambda, \omega)=\frac{e^{\lambda F_{n}(\omega)}}{\prod_{t \in T^{(n)} \backslash\{0\}\{-1\}} E\left[e^{\lambda g_{t}\left(x_{2_{t}}, X_{1_{t}}, X\right)} \mid X_{1_{t}}, X_{2_{t}}\right]},
$$

where $\lambda$ is a real number. Then $\left\{t_{n}(\lambda, \omega), \mathcal{F}_{n}, n \geq 1\right\}$ is a nonnegative martingale.

Proof: The proof of Lemma 1 is similar to Lemma 2.1 in [9], so we omit it.

Theorem 1 Let $\left\{X_{t}, t \in T\right\}$ and $\left\{g_{t}(x, y, z), t \in T\right\}$ be defined as Lemma 1,

$$
G_{n}(\omega)=\sum_{t \in T^{(n)} \backslash\{0\}\{-1\}} E\left[g_{t}\left(X_{2_{t}} X_{1_{t}}, X_{t}\right) \mid X_{1_{t}}, X_{2_{t}}\right],
$$


and $\left\{a_{n}, n \geq 1\right\}$ be a sequence of nonnegative random variables. Let $a>0$. Set

$$
A=\left\{\lim _{n \rightarrow \infty} a_{n}=\infty\right\}
$$

and

$$
\begin{aligned}
D(a)= & \left\{\limsup _{n \rightarrow \infty} \frac{1}{a_{n}} \sum_{t \in T^{(n)} \backslash\{0\}\{-1\}}\right. \\
& \left.E\left[g_{t}^{2}\left(X_{2_{t}}, X_{1_{t}}, X_{t}\right) e^{a \mid g_{t}\left(X_{2_{t}}, X_{1_{t}}, X_{t}\right)} \mid X_{1_{t}}, X_{2_{t}}\right]=M(\omega)<\infty\right\} \cap A .
\end{aligned}
$$

Then

$$
\lim _{n \rightarrow \infty} \frac{1}{a_{n}}\left(F_{n}(\omega)-G_{n}(\omega)\right)=0 \quad \text { a.e. } \quad \omega \in D(a) .
$$

Proof: By Lemma 1, we have known that $\left\{t_{n}(\lambda, \omega), \mathcal{F}_{n}, n \geq 1\right\}$ is a nonnegative martingale. According to Doob martingale convergence theorem, we have

$$
\lim _{n \rightarrow \infty} t_{n}(\lambda, \omega)=t(\lambda, \omega)<\infty \quad \text { a.e. }
$$

By (10) we have

$$
\limsup _{n \rightarrow \infty} \frac{1}{a_{n}} \ln t_{n}(\lambda, \omega) \leq 0 \quad \text { a.e. } \quad \omega \in A .
$$

By (5), (6) and (11), we get

$$
\limsup _{n \rightarrow \infty} \frac{1}{a_{n}}\left\{\lambda F_{n}(\omega)-\sum_{t \in \mathbb{T}^{(n)} \backslash\{0\}\{-1\}} \ln E\left[e^{\lambda g_{t}\left(X_{2 t}, X_{1^{\prime}}, X_{t}\right)} \mid X_{1_{t}}, X_{2_{t}}\right]\right\} \leq 0 \quad \text { a.e. } \omega \in A .
$$

By (12) and inequalities $\ln x \leq x-1(x>0), 0 \leq e^{x}-1-x \leq \frac{x^{2}}{2} e^{|x|}$, as $0<|\lambda| \leq a$ we have

$$
\begin{aligned}
& \limsup _{n \rightarrow \infty} \frac{1}{a_{n}}\left\{\lambda F_{n}(\omega)-\lambda \sum_{t \in T(n) \backslash\{0\}\}-1\}} E\left[g_{t}\left(X_{2^{\prime}}, X_{1^{\prime}}, X_{t}\right) \mid X_{1_{t}}, X_{2_{t}}\right]\right\} \\
& \leq \limsup _{n \rightarrow \infty} \frac{1}{a_{n}}\left\{\sum_{t \in T^{(n)} \backslash\{0\}\{-1\}} \ln E\left[e^{\lambda g_{t}\left(X_{2}, X_{1}, X_{t}\right)} \mid X_{1_{t}}, X_{2_{t}}\right]\right. \\
& \left.-\lambda \sum_{t \in T^{(n)} \backslash\{0\}\{-1\}} E\left[g_{t}\left(X_{2_{t}}, X_{1^{\prime}}, X_{t}\right) \mid X_{1^{\prime}}, X_{2_{t}}\right]\right\} \\
& =\limsup _{n \rightarrow \infty} \frac{1}{a_{n}} \sum_{t \in T^{(n)} \backslash\{\rho\}\{-1\}}\left\{\ln E\left[e^{\lambda g_{t}\left(X_{2 t}, X_{1_{t}}, X_{t}\right)} \mid X_{1_{t}}, X_{2_{t}}\right]-E\left[\lambda g_{t}\left(X_{2_{t}}, X_{1_{t}}, X_{t}\right) \mid X_{1_{t}}, X_{2_{t}}\right]\right\} \\
& \leq \limsup _{n \rightarrow \infty} \frac{1}{a_{n}} \sum_{t \in T^{(n)} \backslash\{\rho\}\{-1\}}\left\{E\left[e^{\lambda g_{t}\left(X_{2_{t}}, X_{1_{t}}, X_{t}\right)} \mid X_{1_{t}}, X_{2_{t}}\right]-1-E\left[\lambda g_{t}\left(X_{2_{t}}, X_{1_{t}}, X_{t}\right) \mid X_{1_{t}}, X_{2_{t}}\right]\right\} \\
& \leq \frac{\lambda^{2}}{2} \limsup _{n \rightarrow \infty} \frac{1}{a_{n}} \sum_{t \in T^{(n)} \backslash\{\rho\}\{-1\}}\left\{E\left[g_{t}^{2}\left(X_{2_{t}}, X_{1_{t}}, X_{t}\right) e^{a \mid g_{t}\left(X_{2_{t}}, X_{1_{t}}, X_{t}\right)} \mid X_{1_{t}}, X_{2_{t}}\right]\right\} \\
& =\frac{\lambda^{2}}{2} M(\omega) \quad \omega \in D(a), \quad \text { a.e. }
\end{aligned}
$$

Letting $0<\lambda \leq a$, by (13), we have

$$
\limsup _{n \rightarrow \infty} \frac{1}{a_{n}}\left\{F_{n}(\omega)-\sum_{t \in T^{(n)} \backslash\{0\}\{-1\}} E\left[g_{t}\left(X_{2_{t}}, X_{1_{t}}, X_{t}\right) \mid X_{1_{t}}, X_{2_{t}}\right]\right\} \leq \frac{\lambda}{2} M(\omega), \quad \omega \in D(a) .
$$


Let $\lambda \rightarrow 0^{+}$in (14), by (8) we have

$$
\limsup _{n \rightarrow \infty} \frac{1}{a_{n}}\left\{F_{n}(\omega)-\sum_{t \in T^{(n)} \backslash\{o\}\{-1\}} E\left[g_{t}\left(X_{2_{t}}, X_{1_{t}}, X_{t}\right) \mid X_{1_{t}}, X_{2_{t}}\right]\right\} \leq 0, \quad \omega \in D(a) .
$$

Taking $-a \leq \lambda<0$ in (15), similarly we get

$$
\limsup _{n \rightarrow \infty} \frac{1}{a_{n}}\left\{F_{n}(\omega)-\sum_{t \in T^{(n)} \backslash\{0\}\{-1\}} E\left[g_{t}\left(X_{2_{t}}, X_{1_{t}}, X_{t}\right) \mid X_{1_{t}}, X_{2_{t}}\right]\right\} \geq 0, \quad \omega \in D(a) .
$$

Combining (7), (15) and (16), we obtain (9).

Corollary 1 Let $\left\{X_{t}, t \in T\right\}$ be a general infinite tree $T$ with uniformly bounded degree defined by Definition 2. Let $\left\{g_{t}(x, y, z), t \in T\right\}$ be a collection of uniformly bounded functions defined on $G^{3}$. That is, there exists $K>0$ such that $\left|g_{t}(x, y, z)\right| \leq K$. $F_{n}(\omega)$ and $G_{n}(\omega)$ be given by (5) and (7), respectively. Let $N \geq 0$, then

$$
\lim _{n \rightarrow \infty} \frac{1}{\left|T^{(n+N)}\right|}\left(F_{n}(\omega)-G_{n}(\omega)\right)=0, \quad \text { a.e. }
$$

Proof: Letting $a_{n}=\left|T^{(n+N)}\right|$ in Theorem 1, noticing that $\left\{g_{t}(x, y), t \in T\right\}$ are uniformly bounded, and then $D(\alpha)=\Omega$ for all $\alpha>0$. This corollary follows from Theorem 1 directly.

In the following, let $I_{k}(x)=\left\{\begin{array}{ll}1 & x=k \\ 0 & x \neq k\end{array}\right.$. We always let $N \geq 0, m, l, k \in G, d^{0}(t):=1$ and denoted by

$$
\begin{aligned}
& d^{N}(t):=\left|\tau \in T: N_{\tau}=t\right| \\
& S_{l, k}^{N}(A):=\sum_{t \in A} I_{l}\left(X_{1_{t}}\right) I_{k}\left(X_{t}\right) d^{N}(t), \\
& S_{m, l, k}^{N}(A):=\sum_{t \in A} I_{m}\left(X_{2_{t}}\right) I_{l}\left(X_{1_{t}}\right) I_{k}\left(X_{t}\right) d^{N}(t) .
\end{aligned}
$$

Corollary 2 Let $T$ be a general infinite tree with uniformly bounded degree. Let $\left\{X_{t}, t\right.$ $\in T\}$ be a $T$-indexed second-order Markov chain with state space $G$ defined by Definition 2. Then for all $m, l, k \in G, N \geq 0$, we have

$$
\lim _{n \rightarrow \infty} \frac{1}{\left|T^{(n+N)}\right|}\left\{S_{l, k}^{N}\left(T^{(n)} \backslash\{-1\}\right)-\sum_{m \in G} S_{m, l}^{N+1}\left(T^{(n-1)} \backslash\{-1\}\right) P(k \mid m, l)\right\}=0 \quad \text { a.e. }
$$

Proof: Because of the uniformly bounded degree of $T$, there is $a<\infty$ with $d^{N}(t)<a^{N}$. Let $g_{t}(x, y, z)=d^{N}(t) I_{l}(y) I_{k}(z), a_{n}=\left|T^{(n+N)}\right|$. Obviously, $\left\{g_{t}(x, y, z), t \in T\right\}$ are uniformly bounded. Since

$$
\begin{aligned}
F_{n}(\omega) & =\sum_{t \in T^{(n)} \backslash\{0\}\{-1\}} g_{t}\left(X_{2_{t}}, X_{1_{t}}, X_{t}\right) \\
& =\sum_{t \in T^{(n)} \backslash\{0\}\{-1\}} I_{l}\left(X_{1_{t}}\right) I_{k}\left(X_{t}\right) d^{N}(t) \\
& =S_{l, k}^{N}\left(T^{(n)} \backslash\{-1\}\right)-I_{l}\left(X_{-1}\right) I_{k}\left(X_{o}\right) d^{N}(o),
\end{aligned}
$$


and

$$
\begin{aligned}
G_{n}(\omega) & =\sum_{t \in T^{(n)} \backslash\{0\}\{-1\}} E\left[g_{t}\left(X_{2_{t}}, X_{1_{t}}, X_{t}\right) \mid X_{1_{t}}, X_{2_{t}}\right] \\
& =\sum_{t \in T^{(n)} \backslash\{0\}\{-1\}} \sum_{x_{t} \in G} g_{i}\left(X_{2_{t}}, X_{1_{t}}, x_{t}\right) P\left(x_{t} \mid X_{1_{t}}, X_{2_{t}}\right) \\
& =\sum_{t \in T^{(n)} \backslash\{0\}\{-1\}} \sum_{x_{t} \in G} I_{l}\left(X_{1_{t}}\right) I_{k}\left(x_{t}\right) d^{N}(t) P\left(x_{t} \mid X_{1_{t}}, X_{2_{t}}\right) \\
& =\sum_{t \in T^{(n)} \backslash\{0\}\{-1\}} I_{l}\left(X_{1_{t}}\right) d^{N}(t) P\left(k \mid X_{1_{t}}, X_{2_{t}}\right) \\
& =\sum_{m \in G} \sum_{t \in T^{(n)} \backslash\{o\}\{-1\}} I_{m}\left(X_{2_{t}}\right) I_{l}\left(X_{1_{t}}\right) d^{N}(t) P(k \mid l, m) \\
& =\sum_{m \in G} \sum_{t \in T^{(n-1)} \backslash\{-1\}} I_{m}\left(X_{1_{t}}\right) I_{l}\left(X_{t}\right) d^{N+1}(t) F(k \mid l, m) \\
& =\sum_{m \in G} S_{m, l}^{N+1}\left(T^{(n-1)} \backslash\{-1\}\right) P(k \mid l, m) .
\end{aligned}
$$

This corollary follows from Corollary 1 directly.

Corollary 3 Let $\left\{X_{t}, t \in T\right\}$ be a second-order Markov chain taking values in $G$ indexed by a general infinite tree with uniformly bounded degree defined as before. Then for all $m, l, k \in G$, we have

$$
\lim _{n \rightarrow \infty} \frac{1}{\left|T^{(n+N)}\right|}\left\{S_{m, l, k}^{N}\left(T^{(n)} \backslash\{o\}\{-1\}\right)-S_{m, l}^{N+1}\left(T^{(n-1)} \backslash\{-1\}\right) P(k \mid m, l)\right\}=0 \text { a.e. }
$$

Proof: Let $g_{t}(x, y, z)=d^{N}(t) I_{m}(x) I_{l}(y) I_{k}(z), a_{n}=\left|T^{(n+N)}\right|$. Obviously, $\left\{g_{t}(x, y, z), t \in T\right\}$ are uniformly bounded. Since

$$
\begin{aligned}
F_{n}(\omega) & =\sum_{t \in T^{(n)} \backslash\{0\}\{-1\}} g_{t}\left(X_{2_{t}}, X_{1_{t}}, X_{t}\right) \\
& =\sum_{t \in T^{(n)} \backslash\{0\}\{-1\}} I_{m}\left(X_{2_{t}}\right) I_{l}\left(X_{1_{t}}\right) I_{k}\left(X_{t}\right) d^{N}(t) \\
& =S_{m, l, k}^{N}\left(T^{(n)} \backslash\{o\}\{-1\}\right),
\end{aligned}
$$

and

$$
\begin{aligned}
G_{n}(\omega) & =\sum_{t \in T^{(n)} \backslash\{o\}\{-1\}} E\left[g_{t}\left(X_{2_{t}}, X_{1_{t}}, X_{t}\right) \mid X_{1_{t}}, X_{2_{t}}\right] \\
& =\sum_{t \in T^{(n)} \backslash\{o\}\{-1\}} \sum_{x_{t} \in G} g_{t}\left(X_{2_{t}}, X_{1_{t}}, x_{t}\right) P\left(x_{t} \mid X_{1_{t}}, X_{2_{t}}\right) \\
& =\sum_{t \in T^{(n)} \backslash\{o\}\{-1\}} \sum_{x_{t} \in G} I_{m}\left(X_{2_{t}}\right) I_{l}\left(X_{1_{t}}\right) I_{k}\left(x_{t}\right) d^{N}(t) P\left(x_{t} \mid X_{1_{t}}, X_{2_{t}}\right) \\
& =\sum_{t \in T^{(n)} \backslash\{o\}\{-1\}} I_{m}\left(X_{2_{t}}\right) I_{l}\left(X_{1_{t}}\right) d^{N}(t) P(k \mid l, m) \\
& =\sum_{t \in T(n-1) \backslash\{-1\}} I_{m}\left(X_{1_{t}}\right) I_{l}\left(X_{t}\right) d^{N+1}(t) P(k \mid l, m) \\
& =S_{m, l}^{N+1}\left(T^{(n-1)} \backslash\{-1\}\right) P(k \mid l, m) .
\end{aligned}
$$

This corollary follows from Corollary 1 directly. 


\section{LLN and Shannon-McMillan theorems}

In this section, we study the strong law of large numbers and the Shannon-McMillan theorems for a second-order Markov chain indexed by a general infinite tree with uniformly bounded degree. In the following, we first give a definition and a lemma.

Definition 3 Let $G=(1,2, \ldots, N)$ be a finite state space, and

$$
P=(p(k \mid l, m)), \quad m, l, k \in G
$$

be a second-order transition matrix. Define a stochastic matrix as follows:

$$
\bar{P}=(p((l, k) \mid(m, i))), \quad(m, i),(l, k) \in G^{2},
$$

where

$$
p((l, k) \mid(m, i))= \begin{cases}p(k \mid l, m) l=i \\ 0 & \text { otherwise. }\end{cases}
$$

Then $\bar{P}$ is called a two-dimensional stochastic matrix determined by the secondorder transition matrix $P$.

Lemma 2 (see [12])

Let $\bar{P}$ be a two-dimensional stochastic matrix determined by the second-order transition matrix $P$. If the elements of $P$ are all positive, i.e.,

$$
P=(p(k \mid l, m)), \quad p(k \mid l, m)>0, m, l, k \in G,
$$

then $\bar{P}$ is ergodic.

Theorem 2 Let $T$ be a general infinite tree with uniformly bounded degree. Let $\left\{X_{t}, t\right.$ $\in T\}$ be a $T$-indexed second-order Markov chain taking values in $G$ generated by $P$. Let the two-dimensional stochastic matrix $\bar{P}$ determined by $P$ be ergodic. Then for all $m, l, k \in G$, we have

$$
\begin{aligned}
& \lim _{n \rightarrow \infty} \frac{S_{l, k}^{N}\left(T^{(n)} \backslash\{-1\}\right)}{\left|T^{(n+N)}\right|}=\pi(l, k) \quad \text { a.e., } \\
& \lim _{n \rightarrow \infty} \frac{S_{m, l, k}^{N}\left(T^{(n)} \backslash\{0\}\{-1\}\right)}{\left|T^{(n+N)}\right|}=\pi(m, l) P(k \mid l, m) \quad \text { a.e., }
\end{aligned}
$$

where $\{\pi(l, k), l, k \in G\}$ is the stationary distribution determined by $\bar{P}$.

Proof: By (21) and (29), we have

$$
\lim _{n \rightarrow \infty}\left\{\frac{S_{l, k}^{N}\left(T^{(n)} \backslash\{-1\}\right)}{\left|T^{(n+N)}\right|}-\sum_{(m, i) \in G^{2}} \frac{S_{m, i}^{N+1}\left(T^{(n-1)} \backslash\{-1\}\right)}{\left|T^{(n+N)}\right|} P((l, k)(m, i))\right\}=0 \text {. a.e. }(32)
$$

We now prove the following equation by induction: Fixed $N \geq 0$ and for all $h \geq 1$, we have

$$
\lim _{n \rightarrow \infty}\left\{\frac{S_{l, k}^{N}\left(T^{(n)} \backslash\{-1\}\right)}{\left|T^{(n+N)}\right|}-\sum_{(m, i) \in G^{2}} \frac{S_{m, i}^{N+h}\left(T^{(n-h)} \backslash\{-1\}\right)}{\left|T^{(n+N)}\right|} P^{h}((l, k)(m, i))\right\}=0 . \quad \text { a.e. }
$$


The case $h=1$ is immediate from the equation (32). The case $h+1$ follows by

$$
\begin{aligned}
\{ & \left.\frac{S_{l, k}^{N}\left(T^{(n)} \backslash\{-1\}\right)}{\left|T^{(n+N)}\right|}-\sum_{(m, i) \in G^{2}} \frac{S_{m, i}^{N+h+1}\left(T^{(n-h-1)} \backslash\{-1\}\right)}{\left|T^{(n+N)}\right|} P^{h+1}((l, k) \mid(m, i))\right\} \\
= & \left\{\frac{S_{l, k}^{N}\left(T^{(n)} \backslash\{-1\}\right)}{\left|T^{(n+N)}\right|}-\sum_{(m, i) \in G^{2}} \sum_{(s, t) \in G^{2}} \frac{S_{m, i}^{N+h+1}\left(T^{(n-h-1)} \backslash\{-1\}\right)}{\left|T^{(n+N)}\right|} P^{h}((l, k)-(s, t)) P((s, t) \mid(m, i))\right\} \\
= & \left\{\frac{S_{l, k}^{N}\left(T^{(n)} \backslash\{-1\}\right)}{\left|T^{(n+N)}\right|}-\sum_{(s, t) \in G^{2}} \frac{S_{s, t}^{N+h}\left(T^{(n-h)} \backslash\{-1\}\right)}{\left|T^{(n+N)}\right|} P^{h}((l, k) \mid(s, t))\right\} \\
& +\left\{\sum _ { ( s , t ) \in G ^ { 2 } } \left[\frac{S_{s, t}^{N+h}\left(T^{(n-h)} \backslash\{-1\}\right)}{\left|T^{(n+N)}\right|}\right.\right. \\
& \left.\left.-\sum_{(m, i) \in G^{2}} \frac{S_{m, i}^{N+h+1}\left(T^{(n-h-1)} \backslash\{-1\}\right)}{\left|T^{(n+N)}\right|} P((s, t) \mid(m, i))\right] P^{h}((l, k) \mid(s, t))\right\}
\end{aligned}
$$

Notice that the first term vanishes as $n \rightarrow \infty$, because of the induction hypothesis. According to the equation (32), the second term approximates to zero as $n \rightarrow \infty$. By induction, we have (33) holds for all $h \geq 1$. Since $\sum_{(m, i) \in G^{2}} S_{m, l}^{h+N}\left(T^{(n-h)} \backslash\{-1\}\right)=\left|T^{(n+N)}\right|-\left|T^{(h+N-1)}\right|$. By (33), we have

$$
\begin{aligned}
& \limsup _{n \rightarrow \infty}\left|\frac{S_{l, k}^{N}\left(T^{(n)} \backslash\{-1\}\right)}{\left|T^{(n+N)}\right|}-\pi(l, k)\right| \\
& \quad \leq \limsup _{n \rightarrow \infty}\left|\sum_{(m, i) \in G^{2}} \frac{S_{m, i}^{N+h}\left(T^{(n-h)} \backslash\{-1\}\right)}{\left|T^{(n+N)}\right|} P^{h}((l, k) \mid(m, i))-\pi(l, k)\right| \\
& =\limsup _{n \rightarrow \infty}\left|\sum_{(m, i) \in G^{2}} \frac{S_{m, i}^{N+h}\left(T^{(n-h)} \backslash\{-1\}\right)}{\left|T^{(n+N)}\right|}\left(P^{h}((l, k) \mid(m, i))-\pi(l, k)\right)-\frac{\left|T^{(N+h-1)}\right|}{\left|T^{(n+N)}\right|} \pi(l, k)\right| \\
& \left.\leq \limsup _{n \rightarrow \infty} \sum_{(m, i) \in G^{2}} \frac{S_{m, i}^{N+h}\left(T^{(n-h)} \backslash\{-1\}\right)}{\left|T^{(n+N)}\right|} \mid P^{h}((l, k) \mid(m, i))-\pi(l, k)\right)+\limsup _{n \rightarrow \infty} \frac{\left|T^{(N+h-1)}\right|}{\left|T^{(n+N)}\right|} \pi(l, k) \\
& \leq \sum_{(m, i) \in G^{2}}\left|P^{h}((l, k) \mid(m, i))-\pi(l, k)\right| \quad \text { a.e. }
\end{aligned}
$$

Since

$$
\lim _{n \rightarrow \infty} P^{h}((l, k) \mid(m, i))=\pi(l, k), \quad \forall(m, i) \in G^{2},
$$

(30) follows from (35) and (36), (31) follows from (30) and (24).

Corollary 4 Under the conditions of Theorem 2, let $S_{m, l, k}\left(T^{(n)} \backslash\{o\}\{-1\}\right):=S_{m, l, k}^{0}\left(T^{(n)} \backslash\{o\}\{-1\}=\left|\left\{t \in T^{(n)}:\left(X_{2_{t}}, X_{1_{t}}, X_{t}\right)=(m, l, k)\right\}\right|\right.$. Then

$$
\lim _{n \rightarrow \infty} \frac{S_{m, l, k}\left(T^{(n)} \backslash\{o\}\{-1\}\right)}{\left|T^{(n)}\right|}=\pi(m, l) P(k \mid m, l) \quad \text { a.e. }
$$

Let $T$ be a tree, $\left\{X_{t}, t \in T\right\}$ be a stochastic process indexed by tree $T$ taking values in G, $x^{T^{(n)}}$ be the realization of $X^{T^{(n)}}$. Denote

$$
P\left(x^{T^{(n)}}\right)=P\left(X^{T^{(n)}}=x^{T^{(n)}}\right) .
$$

Let

$$
f_{n}(\omega)=-\frac{1}{\left|T^{(n)}\right|} \ln P\left(X^{T^{(n)}}\right),
$$


$f_{n}(\omega)$ is called the entropy density of $X^{T^{(n)}}$. If $\left\{X_{t}, t \in T\right\}$ is a second-order Markov chain indexed by a general infinite tree with uniformly bounded tree defined as in Definition 2, we easily have

$$
f_{n}(\omega)=\frac{1}{\left|T^{(n)}\right|}\left[\ln P\left(X_{-1}, X_{o}\right)+\sum_{t \in T^{(n)} \backslash\{o\}\{-1\}} \ln P\left(X_{t} \mid X_{1^{\prime}}, X_{2_{t}}\right)\right] .
$$

The convergence of $f_{n}(\omega)$ to a constant in a sense ( $L_{1}$ convergence, convergence in probability, a.e. convergence) is called the Shannon-McMillan theorem, or the entropy theorem or the AEP in information theory. Here from Theorems 1, 2, and Corollary 4, we can easily obtain the Shannon-McMillan theorem with a.e. convergence for a second-order Markov chain indexed by a general infinite tree with uniformly bounded tree.

Theorem 3 Let $\left\{X_{t}, t \in T\right\}$ be a second-order Markov chain taking values in $G$ indexed by a general infinite tree with uniformly bounded tree $T$, and $f_{n}(\omega)$ be defined as (38). Then

$$
\lim _{n \rightarrow \infty} f_{n}(\omega)=-\sum_{m \in G} \sum_{l \in G} \sum_{k \in G} \pi(m, l) P(k \mid l, m) \ln P(k \mid l, m) \quad \text { a.e. }
$$

Proof:

$$
\begin{aligned}
\lim _{n \rightarrow \infty} f_{n}(\omega) & =-\lim _{n \rightarrow \infty} \frac{1}{\left|T^{(n)}\right|}\left[\ln P\left(X_{-1}, X_{o}\right)+\sum_{t \in T^{(n)} \backslash\{0\}\{-1\}} \ln P\left(X_{t} \mid X_{1_{t}}, X_{2_{t}}\right)\right] \\
& =-\lim _{n \rightarrow \infty} \frac{1}{\left|T^{(n)}\right|} \sum_{t \in T^{(n)} \backslash\{o\}\{-1\}} \ln P\left(X_{t} \mid X_{1_{t}}, X_{2_{t}}\right) \\
& =-\sum_{m \in G} \sum_{l \in G} \sum_{k \in G} \lim _{n \rightarrow \infty} \frac{1}{\left|T^{(n)}\right|} \sum_{t \in T^{(n)} \backslash\{0\}\{-1\}} I_{m}\left(X_{2_{t}}\right) I_{l}\left(X_{1_{t}}\right) I_{k}\left(X_{t}\right) \ln P(k \mid l, m) \\
& =-\sum_{m \in G} \sum_{l \in G} \sum_{k \in G} \lim _{n \rightarrow \infty} \frac{S_{m, l, k}\left(T^{(n)} \backslash\{o\}\{-1\}\right)}{\left|T^{(n)}\right|} \ln P(k \mid l, m),
\end{aligned}
$$

Equation (39) follows from (40) and (37) directly.

\section{Acknowledgements}

The authors would like to thank the referees for their valuable suggestions and comments. This work is supported by the Research Foundation for Advanced Talents of Jiangsu University(11JDG116) and the National Natural Science Foundation of China (11071104, 11171135, 71073072).

\section{Author details}

${ }^{1}$ Faculty of Science, Jiangsu University, Zhenjiang, 212013, China ${ }^{2}$ School of Mathematical Science, Huaiyin Normal University, Huaian, 223300, China

\section{Authors' contributions}

ZS, WY and LT carried out the design of the study and performed the analysis. QZ participated in its design and coordination. All authors read and approved the final manuscript.

\section{Competing interests}

The authors declare that they have no competing interests.

Received: 15 June 2011 Accepted: 4 January 2012 Published: 4 January 2012

References

1. Benjamini, I, Peres, Y: Markov chains indexed by trees. Ann Probab. 22, 219-243 (1994). doi:10.1214/aop/1176988857

2. Berger, T, Ye, Z: Entropic aspects of random fields on trees. IEEE Trans Inform Theory. 36, 1006-1018 (1990). doi:10.1109/ 18.57200 
3. Ye, Z, Berger, T: Ergodic, regularity and asymptotic equipartition property of random fields on trees. Comb Inform Syst Sci. 21, 157-184 (1996)

4. Ye, Z, Berger, T: Information Measure for Discrete Random Field. Science, Beijing. (1998)

5. Pemantle, R: Andomorphism invariant measure on tree. Ann Probab. 20, 1549-1566 (1992). doi:10.1214/aop/1176989706

6. Yang, WG, Liu, W: Strong law of large numbers for Markov chains fields on a bethe tree. Statist Probab Lett. 49 245-250 (2000). doi:10.1016/50167-7152(00)00053-5

7. Yang, WG: Some limit properties for Markov chains indexed by a homogeneous tree. Statist Probab Lett. 65, 241-250 (2003). doi:10.1016/j.spl.2003.04.001

8. Takacs, C: Strong law of large numbers for branching Markov chains. Markov Process. Relat Fields. 8, 107-116 (2001)

9. Yang, WG, Ye, Z: The Asymptotic Equipartition property for Markov chains indexed by a Homogeneous tree. IEEE Trans Inform Theory. 53(9), 3275-3280 (2007)

10. Huang, HL, Yang, WG: Strong law of large numbers for Markov chains indexed by an infinite tree with uniformly bounded degree. Sci China. 51(2), 195-202 (2008). doi:10.1007/s11425-008-0015-1

11. Shi, ZY, Yang, WG: Some limit properties of random transition probability for second-order nonhomogeneous Markov chains indexed by a tree. J Inequal Appl (2009). ID 503203

12. Yang, WG, Liu, W: The asymptotic equipartition property for Mth-order nonhomogeneous Markov information sources IEEE Trans Inf Theory. 50(12), 3326-3330 (2004). doi:10.1109/TIT.2004.838339

doi:10.1186/1029-242X-2012-2

Cite this article as: Shi et al: Some limit theorems for the second-order Markov chains indexed by a general infinite tree with uniform bounded degree. Journal of Inequalities and Applications 2012 2012:2.

\section{Submit your manuscript to a SpringerOpen ${ }^{\circ}$} journal and benefit from:

- Convenient online submission

- Rigorous peer review

- Immediate publication on acceptance

- Open access: articles freely available online

- High visibility within the field

- Retaining the copyright to your article

Submit your next manuscript at $\gg$ springeropen.com 